\title{
Homogeneous nucleation of colloidal melts under the influence of shearing fields
}

\author{
Ronald Blaak $^{1}$, Stefan Auer ${ }^{2}$, Daan Frenkel ${ }^{3}$ and Hartmut Löwen ${ }^{1}$ \\ ${ }^{1}$ Institut für Theoretische Physik II, Heinrich-Heine-Universität, Universitätsstraße 1, \\ D-40225 Düsseldorf, Germany \\ 2 Department of Chemistry, Cambridge University, Lensfield Road, Cambridge CB2 1EW, UK \\ ${ }^{3}$ FOM Institute for Atomic and Molecular Physics, Kruislaan 407, 1098 SJ Amsterdam, \\ The Netherlands
}

Received 28 March 2004

Published 10 September 2004

Online at stacks.iop.org/JPhysCM/16/S3873

doi:10.1088/0953-8984/16/38/007

\begin{abstract}
We study the effect of shear flow on homogeneous crystal nucleation, using Brownian dynamics simulations in combination with an umbrella samplinglike technique. The symmetry breaking due to shear results in anisotropic radial distribution functions. The homogeneous shear rate suppresses crystal nucleation and leads to an increase of the size of the critical nucleus. These observations can be described by a simple, phenomenological extension of classical nucleation theory. In addition, we find that nuclei have a preferential orientation with respect to the direction of shear. On average the longest dimension of a nucleus is along the vorticity direction, while the shortest dimension is preferably perpendicular to that and slightly tilted with respect to the gradient direction.
\end{abstract}

(Some figures in this article are in colour only in the electronic version)

\section{Introduction}

It is well known that fluids can be cooled to temperatures below the coexistence temperature of the liquid and solid phases. Density fluctuations in this supercooled regime lead to the continuous formation of small crystalline nuclei. Even though the crystalline phase is the thermodynamically stable phase, a supercooled system can remain for longer times in a liquidlike form. The reason lies in the fact that there is a force counteracting crystallization which has its origin in the interface that needs to be formed between small crystallites and the surrounding liquid. This results in a nucleation barrier which needs to be overcome in order to solidify the system. This intuitive picture forms the basis of what at present is known as classical nucleation theory $[1,2]$.

Understanding of the underlying principles of nucleation and the growth to macroscopic sizes finds applications in many areas, e.g. protein crystallization and metallurgy [3-5]. The 
first experimental studies of crystal nucleation were light scattering experiments [6-8] on hard sphere colloids, which form a well understood model system [9]. Crystallization rates were measured by Gasser et al [10] using confocal microscopy in weakly charged colloids. Recently computer simulations have been used to explore nucleation phenomena and by using special simulation techniques it was possible to obtain quantitative results on the absolute rate of crystal nucleation in colloidal suspensions $[11,12]$ and compare them with experiments.

In the present work we focus on nucleation outside equilibrium, i.e. in the presence of a homogeneous shear field. Experiments on crystallization under shear suggest two different scenarios. In some cases one observes that crystallization is enhanced by the presence of shear [13-16]. This can, at least qualitatively, be understood, on the basis of the idea that shear might facilitate the formation of layers in the system, making it easier to crystallize. Other experiments report that crystallization is suppressed $[17,18]$. In the later case one could imagine that the shear will detach particles from newly born crystallites. Both effects are also found in heterogeneous nucleation under shear in single- or double-wall confinement. For low and moderate shear rates two different types of layering sliding are observed, while for high shear rates the layers are found to destabilize [19].

A priori it is not clear which of the two scenarios will dominate and how this depends on the external conditions. In simulations by Butler and Harrowell [20] for the crystallization kinetics of particles with Yukawa interaction subjected to shear rates, it was observed that crystallization is suppressed by shear. In the present work we will focus on nucleation in its bare bones and extract information on the properties of nuclei that are formed in a supercooled melt. A preliminary account of our simulation results has already been published [21]. We include here further data on pair correlations and the critical nucleus structure and orientation, and describe the simulation technique in more detail.

The remainder of this paper is organized as follows. In section 2 we introduce the model and explain the combination of different techniques that we used for our simulations. The simulation results for the pair correlation functions and the size distribution function of the nuclei are shown in section 3. In section 4 we make a simple, phenomenological extension of classical nucleation theory and compare this with the results obtained from the simulations. A more detailed analysis of the shape and relative orientation with respect to the shear direction is performed in section 5. We finish in section 6 with a summary and a discussion of our main results.

\section{The model}

In order to simulate homogeneous crystal nucleation under shear, we have chosen to consider a simple model of charge-stabilized colloidal suspensions subjected to a linear shear flow. Due to screening of the micro-ions, these particles mutually interact effectively via a repulsive Yukawa potential [9]

$$
V(r)=\epsilon \frac{\mathrm{e}^{-\kappa r}}{\kappa r},
$$

where $r$ is the distance between the centres of the colloidal particles. The magnitude of the screening is expressed by the inverse screening length $\kappa$ and the strength of the interaction is denoted by $\epsilon$.

In addition to the normal inter-particle interaction, we also want to apply shear to the system. This means that we are dealing with a non-equilibrium situation; hence we need to make use of dynamical simulations rather than simulations of the Monte Carlo type. For low volume fractions of charged suspensions one can neglect the hydrodynamic interactions 
between the colloids, which enables us to apply Brownian dynamics $[22,23]$. In this overdamped motion, it is assumed that particles move at constant velocity proportional to the force due to other particles, and that the solvent exerts a random force with a Gaussian distribution. The steady shear rate $\dot{\gamma}$ imposed on the system is assumed to generate a linear velocity gradient in the solvent, which in turn results in a linear force field for the colloids. The combination of Brownian dynamics and shear results in the following equations of motion:

$$
\vec{r}_{i}(t+\delta t)=\vec{r}_{i}(t)+\delta t \frac{\vec{f}_{i}(t)}{\xi}+\delta \vec{r}^{\mathrm{G}}+\delta t \dot{\gamma} y_{i}(t) \hat{x} .
$$

Here $\vec{r}_{i}(t)=\left(x_{i}(t), y_{i}(t), z_{i}(t)\right)$ is the position of the $i$ th colloidal particle at time $t$. In a small time interval $\delta t$ this particle moves under the influence of the sum of the conservative forces $\vec{f}_{i}(t)$ arising from the pair interaction (1) of particle $i$ with the neighbouring particles. The friction constant $\xi$ with the solvent is related to the short time diffusion constant $D$ by $\xi=k_{\mathrm{B}} T / D$. The stochastic displacements are independently drawn from a Gaussian distribution with zero mean and variance $\left\langle\left(\delta r_{i \alpha}^{\mathrm{G}}\right)^{2}\right\rangle=2 D \delta t$, where $\alpha$ stands for one of the Cartesian components. The last term in equation (2) represents the applied shear in the $x$ direction, and imposes an explicit linear flow field.

All simulations reported here are done for a system of 3375 colloidal particles and the interaction strength is fixed to $\epsilon=1.48 \times 10^{4} k_{\mathrm{B}} T$. For practical purposes the interaction potential is truncated at the cut-off distance $r_{\mathrm{c}}=10 / \kappa$, by shifting the potential to zero. The inter-particle forces are not affected by this procedure. For simplicity we use a cubic simulation box to which we apply the shifted periodic boundary conditions. These were introduced by Lees and Edwards [24] and are required to deal with the presence of shear.

The simple combination of Brownian dynamics with a linear imposed velocity field and Lees-Edwards boundary conditions forms the heart of the simulations. This ensures that the starting configurations follow the proper equations of motion. To facilitate the connection with Gibbs free energies, however, it is desirable to do the simulations at constant osmotic pressure. Hereto we have added volume moves as normally used in equilibrium Monte Carlo simulations of the isobaric ensemble. In practice this means that after a large number of Brownian dynamics steps, the simulation box expands or shrinks isotropically. The difference in potential energy due to this volume move is used in order to accept or reject this move. The value of the applied pressure is chosen such that the bulk behaviour of the system is in the supercooled regime. Therefore the prepared system will remain liquid, even though the crystalline phase is more stable. Due to fluctuations inside such a liquid, small nuclei are continuously formed and dissolved. Only once a sufficiently large nucleus is formed, exceeding the critical nucleus size, will the growth of the nucleus dominate and lead to a full crystallization of the system.

The quantity of interest of our simulations is the cluster size distribution function $P(n)$, which describes the probability of finding a nucleus of $n$ particles. This is a well defined object, even in the non-equilibrium situation of the presence of shear, provided that after a sufficiently long simulation time the system reaches a steady state situation. To this end we need to analyse the configurations obtained by the simulations and detect all the nuclei in the liquid. This can be done with the aid of bond orientational order parameters introduced by Steinhardt et al [25] and applied to study crystal nucleation by Frenkel and co-workers [26-28]. All particles within a short distance of the particle of interest are determined, and the vectors connecting them are resolved in polar angles. These can be used to determine rotationally invariant order parameters, which characterize the neighbourhood of a particle, and allow us to distinguish a local fluid environment from a crystalline environment. It is even possible to distinguish between different crystalline structures. Solid particles that are in each other's 
neighbourhoods belong to the same cluster. In this manner we obtain the total number of clusters in the system and each of their sizes.

By performing long simulations, in which between $10^{6}$ and $10^{7}$ Brownian dynamics steps are made, we can now measure the probability size distribution function $P(n)$ in a simple way. After 20 Brownian dynamics steps and possibly a volume move (on average one per hundred BD steps), we analyse the configuration in determining the size and the number of clusters present in the liquid. These are used to make a histogram of the possible cluster sizes, and the frequency is proportional to the probability of observing such a cluster size. Note that even in the presence of shear, and therefore in non-equilibrium, this procedure can be followed.

Nucleation, however, is a rare process and, unless the system has a large nucleation rate [29], no sufficient statistics can be obtained by merely the technique described above. The solution to this problem is the usage of umbrella sampling [30]. This technique stems from equilibrium Monte Carlo simulations and introduces an additional potential to the system. Such a bias should depend on a relevant reaction coordinate of the system under consideration and is able to restrain the system in a meta-stable situation. The traditional interpretation is that the bias is used as an external potential, leading to a biased ensemble. It therefore might seem somewhat surprising that the technique can be used even in non-equilibrium situations. The reason for this apparent ambiguity is that umbrella sampling can be considered as a plain mathematical trick.

In order to measure the steady state probability distribution $P(n)$, we need to sample the relative probabilities of $P(n+1) / P(n)$, which is achieved by growing paths by Brownian dynamics simulation from one cluster size to another. In principle this could be done by starting with a chosen cluster size $n$ and counting the number of times the cluster grows or shrinks by one particle or remains at the same size in a small interval of time, and averaging afterwards over many different realizations of the initial configuration. The number of counts of a given size $n$ is then proportional to $P(n)$. The obvious problem is that below the critical nucleus size the cluster will mainly shrink, and beyond the critical size it will mainly grow. This would be acceptable, but causes a different problem, i.e. how to obtain many configurations of a given size which have a very low probability.

This problem is circumvented by using the bias. For each simulation we use a fixed biasing function based on some preferred cluster size $n_{0}$, which has the simple form

$$
V_{\text {bias }}(n)=\frac{1}{2} \alpha\left(n-n_{0}\right)^{2}
$$

with $\alpha$ a measure for the strength of restraint. We start with a given configuration and follow the normal Brownian dynamics as described above. After a fixed time interval we measure the size $n$ of the largest cluster. We now continue with either the final configuration or the previous starting configuration as the new initial configuration. Only this is determined by the bias that we introduce; it therefore has no effect on the dynamics of the system and is just an aid for generating new initial configurations. By choosing the bias appropriate to a harmonic-like form, we ensure that the probabilities of growing a bigger cluster or shrinking to a smaller cluster with respect to $n_{0}$ are approximately the same. The count of the number of clusters of a given size is now proportional to the ratio of $P(n)$ and $P_{\text {bias }}(n)$ for the known bias that we impose. By performing simulations for different preferred sizes $n_{0}$, we obtain successive parts of the function $P(n)$ which can be combined to form the complete function.

\section{Simulation results}

The pressures at which simulations are performed are such that the equilibrium bulk phase without shear is a face-centred-cubic crystalline phase [12,31]. Hence the liquid state in which 


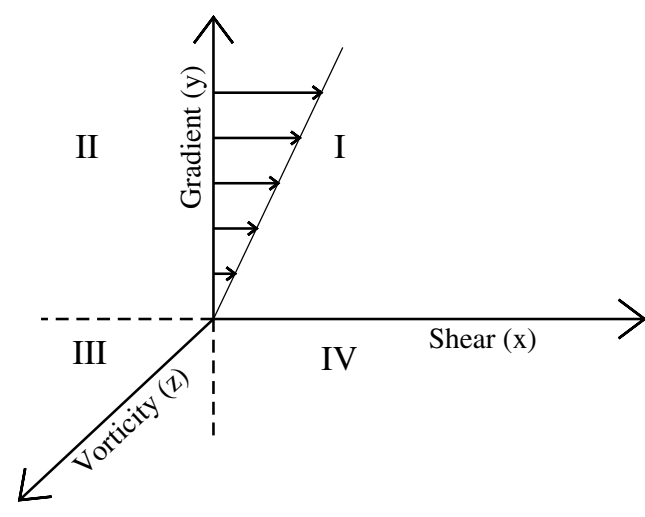

Figure 1. A schematic drawing of the different directions in the problem. The Roman numerals label the different quadrants of space.

we prepare the system is only meta-stable. Nevertheless, the pressure is still small enough that spontaneous crystallization is not observed within the duration of the simulation and even in the absence of the bias the system remains liquid and only forms nuclei smaller than the critical nucleus size.

One of the consequences of the presence of shear is that this introduces a symmetry breaking in the fluid. This can be seen easily in the two-particle distribution function $g^{(2)}\left(\vec{r}_{1}, \vec{r}_{2}\right)$. Whereas in bulk equilibrium this function is only dependent on the absolute distance $\left|\overrightarrow{r_{1}}-\overrightarrow{r_{2}}\right|$, in the presence of shear the function will also depend on the relative direction. In general the function can be expanded in a series of modified spherical harmonics $C_{l, m}(\theta, \phi)$ with distance dependent coefficients $g_{l, m}(r)$ :

$$
g(r, \theta, \phi)=\sum_{l=0}^{\infty} \sum_{m=-l}^{l} \frac{2 l+1}{4 \pi} g_{l, m}(r) C_{l, m}(\theta, \phi),
$$

where $\theta$ is the angle with respect to the vorticity direction, i.e. $\cos \theta=\hat{r} \cdot \hat{z}$, and $\phi$ the angle with the shear direction, i.e. $\cos \phi=\hat{r} \cdot \hat{z} / \sqrt{1-(\hat{r} \cdot \hat{z})^{2}}$ (see figure 1). Note that without the symmetry breaking due to shear, only the isotropic contribution remains, i.e. the usual radial distribution function $g(r) \equiv g_{0,0}(r)$.

The distance dependent coefficients follow directly from the orthogonality relation of the spherical harmonics:

$$
g_{l, m}(r)=\int \mathrm{d} \cos \theta \int \mathrm{d} \phi g(r, \theta, \phi) C_{l, m}^{*}(\theta, \phi)
$$

and can easily be obtained from simulations. Due to symmetries present in the system and the suitably chosen reference frame, only functions with both indices $l$ and $m$ even have non-zero contributions. It turns out that the first-order correction to the radial distribution function is dominated by the imaginary $\operatorname{part} \operatorname{Im}\left(g_{2,2}(r)\right)$ of the function $g_{2,2}(r)$.

In figure 2 we show the ratio of $\operatorname{Im}\left(g_{2,2}(r)\right)$ and the function $g_{0,0}(r)$ as obtained from simulations without bias and for different shear rates. The corresponding function, which was statistically averaged, is $\operatorname{Im}\left(C_{2,2}\right)=-\frac{1}{4} \sqrt{3} \sin ^{2} \theta \sin 2 \phi$. This quantity is able to distinguish whether a particle is preferably found in the first (third) or in the second (fourth) quadrant of space provided that another particle is in the origin (see figure 1). The signal is phase shifted with respect to the oscillations in the radial distribution function, such that particle distances in a peak of a distribution function and located in the first quadrant are typically closer than for those particles found in the second quadrant. It can also be observed that the amplitude grows linearly with the shear rate. The distribution functions $g_{0,0}(r)$ are, within the accuracy of the simulations, identical for these moderate shear rates. 

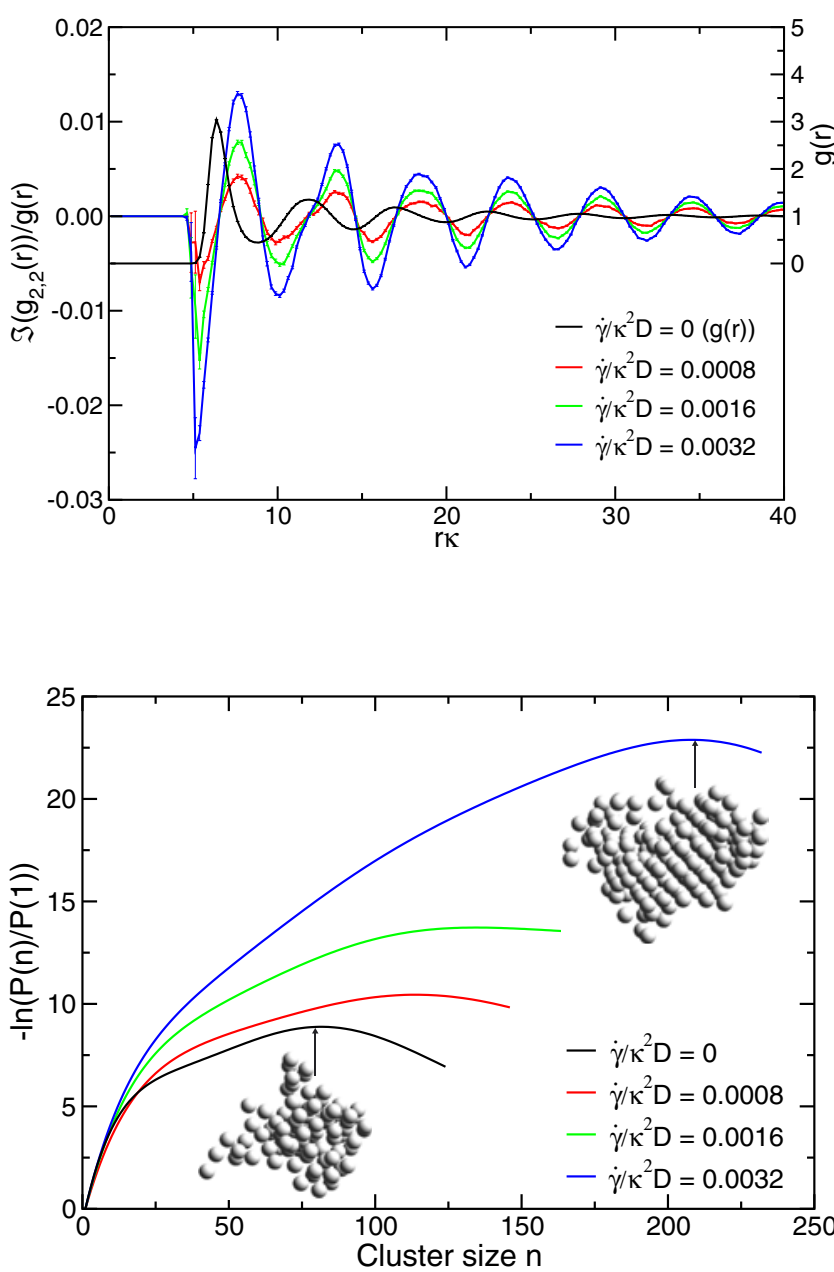

Figure 2. The imaginary part of the function $g_{2,2}(r)$ as a function of the distance for fixed pressure $\beta P / \kappa^{3}=$ 0.240 and different shear rates. For comparison, the radial distribution function $g(r)$ is included.
Figure 3. The negative logarithm of the probability $P(n)$ of finding a cluster of $n$ solid-like particles normalized by $P(1)$ for pressure $\beta P / \kappa^{3}=0.24$ and different applied shear rates, from bottom to top: $\dot{\gamma} /\left(\kappa^{2} D\right)=0,0.8 \times 10^{-3}, 1.6 \times 10^{-3}$ and $3.2 \times 10^{-3}$. The insets show typical snapshots of critical nuclei for the largest shear rate and the zeroshear case.

In order to obtain the probability function $P(n)$ for observing a cluster of $n$ particles, we perform simulations for fixed pressure and shear rate. The values of the preferred size $n_{0}$ in the bias (3) are chosen in multiples of 10 and the strength $\alpha=0.15$. This ensures that the windows of observed cluster sizes are sufficiently wide to produce overlap between neighbouring windows. When the bias is included, we not only find a single big nucleus, but also find that small nuclei can be observed. It suffices, however, to count just the largest cluster, as it contains those fluctuations in size that determine the probability function $P(n)$. The overlap between neighbouring windows is used to match successive parts of $P(n)$. The only exception is the first part of the function, where small nuclei are important. For that region an unbiased simulation is used in which all clusters in the system are used. For a more detailed discussion on how the curve is obtained we refer the reader to [28].

The results of the simulations for a single pressure and several shear rates are shown in figure 3. It shows the negative logarithm of the relative probability function $P(n)$ normalized with the probability $P(1)$ of finding a cluster of only a single particle. For increasing values of the shear rate, the maximum in the curves shifts towards larger values and larger cluster sizes. 


\section{Extension of classical nucleation theory}

Classical nucleation theory links the probability of finding a cluster of given size to the Gibbs free energy and allows one to interpret a probability function as shown in figure 3 for the zero-shear case in terms of a nucleation barrier [28]. It splits the cost in Gibbs free energy $\Delta G$ for creating a spherical nucleus in a liquid into two contributions:

$$
\Delta G=-\frac{4}{3} \pi R^{3} \rho_{\mathrm{S}}|\Delta \mu|+4 \pi R^{2} \gamma_{\mathrm{SL}} .
$$

On the one hand, there is a gain in energy proportional to the volume of the nucleus with radius $R$ due to the difference in chemical potential $\Delta \mu$ between the solid with density $\rho_{\mathrm{S}}$ and the liquid phase. On the other hand, we have a loss in energy, since an interface between the solid nucleus and surrounding liquid needs to be formed, described by $\gamma_{\mathrm{SL}}$, the interfacial free energy.

It can be shown that the free energy cost of creating a nucleus is related to the probability function $P(n)$, and if the appropriate correction is included [28], the height of the maximum in the curve corresponds to the nucleation barrier, while the corresponding cluster size is the size of the critical nucleus. The actual shape of the barrier, as found in simulations, will depend on the way in which a cluster is defined. This will therefore also influence the number of particles in the critical nucleus. The nucleation barrier itself, however, is much less dependent on the cluster definition. In the presence of shear the curves have a similar shape. Also here we can identify the top with a critical nucleus size, since for smaller clusters there is tendency to shrink, while for larger clusters the growth will dominate. The height of the top is a measure for the probability that such a cluster will be formed and is therefore related to the time one needs to wait in order to observe such a cluster size.

It is therefore tempting to also interpret the other probability functions of figure 3 in terms of nucleation barriers. However, due to the presence of shear in the system, we are dealing with a non-equilibrium situation, and although the concept of a nucleation barrier in this case seems intuitively clear, strictly speaking it is wrong since the Gibbs free energy is ill defined outside equilibrium. Surprisingly, the application of statistical mechanics to a non-equilibrium situation can be useful (see, e.g., [32]) and it is a canonical challenge to check the validity of carrying over equilibrium concepts into non-equilibrium physics. With all the principle caveats which one should have in mind about this, we will here assume that we can extend the ideas of classical nucleation theory to the present situation by including shear.

As is the case in classical nucleation theory, we assume that also in the presence of shear a spherical nucleus is formed. The two terms in equation (6) will therefore remain. It is however reasonable to expect that the difference in chemical potential $\Delta \mu$ and interfacial free energy $\gamma_{\text {SL }}$ will be affected by the shear, but that for moderate shear rates an expansion in powers of the shear rate for both of these quantities about their equilibrium values can be performed:

$$
\begin{aligned}
& \Delta \mu=\Delta \mu^{(\mathrm{eq})}\left(1+c_{0} \dot{\gamma}^{2}+\mathcal{O}\left(\dot{\gamma}^{4}\right)\right), \\
& \gamma_{\mathrm{SL}}=\gamma_{\mathrm{SL}}^{(\mathrm{eq})}\left(1+\kappa_{0} \dot{\gamma}^{2}+\mathcal{O}\left(\dot{\gamma}^{4}\right)\right),
\end{aligned}
$$

where due to the invariance of the shear direction only even powers in the shear rate $\dot{\gamma}$ need to be considered. If we insert the expansions (7) in the expression from classical nucleation theory (6) we obtain simple expressions for $\Delta G^{*}$, the height of the nucleation barrier, and $N^{*}$, the size of the critical nucleus:

$$
\begin{aligned}
& \Delta G^{*}=\frac{16 \pi\left[\gamma_{\mathrm{SL}}^{(\mathrm{eq})}\right]^{3}}{3 \rho_{\mathrm{S}}^{2}\left|\Delta \mu^{(\mathrm{eq})}\right|^{2}}\left[1+\left(3 \kappa_{0}-2 c_{0}\right) \dot{\gamma}^{2}+\mathcal{O}\left(\dot{\gamma}^{4}\right)\right], \\
& N^{*}=\frac{32 \pi\left[\gamma_{\mathrm{SL}}^{(\mathrm{eq})}\right]^{3}}{3 \rho_{\mathrm{S}}^{2}\left|\Delta \mu^{(\mathrm{eq})}\right|^{3}}\left[1+\left(3 \kappa_{0}-3 c_{0}\right) \dot{\gamma}^{2}+\mathcal{O}\left(\dot{\gamma}^{4}\right)\right],
\end{aligned}
$$



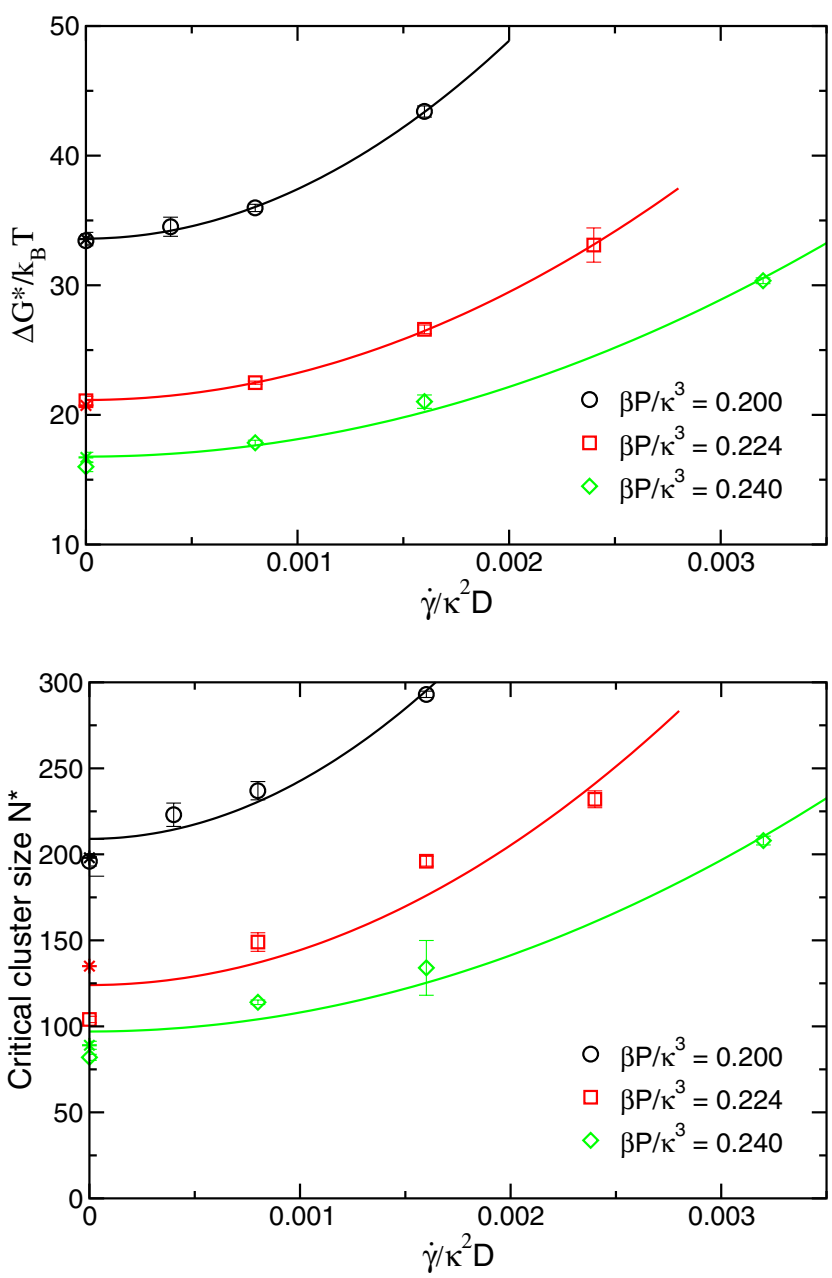

Figure 4. The height of the nucleation barrier $\beta \Delta G^{*}$ as a function of the dimensionless shear rate $\dot{\gamma} / \kappa^{2} D$ for different pressures $P$. The solid curves are parabolic fits through the data. The stars are data from equilibrium Monte Carlo simulations without shear.

Figure 5. The number of particles $N^{*}$ of the critical nucleus as a function of the dimensionless shear rate $\dot{\gamma} / \kappa^{2} D$ and for different pressures $P$. The solid curves are parabolic fits through the data. The stars are data from equilibrium Monte Carlo simulations without shear.

where the corrections with respect to the unsheared case scale to leading order with $\dot{\gamma}^{2}$.

In figures 4 and 5 we show the results from our simulations where we extracted the height of the nucleation barrier and the size of the critical nucleus for various pressures and shear rates. For the zero-shear case, the data from equilibrium Monte Carlo simulations have been included. Parabolic fits through the data support the quadratic dependence of these quantities on the shear rate and also enable us to make estimations for the second-order coefficients in equation (7), the results of which are summarized in table 1 . The presence of shear destabilizes the solid as is indicated by the negative value of $c_{0}$.

The shift of the maximum of the curves in figure 3, as a function of the applied shear rate, is interpreted as an increase in the nucleation barrier and the critical nucleation size. This indicates that the interaction of the system under consideration corresponds to a situation in which crystal nucleation is suppressed by the presence of shear [20]. In addition, the application of shear allows us to supercool the system beyond the point at which in an equilibrium simulation spontaneous crystallization appears. Due to system size restrictions, much larger shear rates could not be accessed, because the increase of the critical nucleus size would lead to simulation artefacts, i.e. a percolating cluster. One can envisage much larger shear rates enhancing crystallization via pre-layering of the system [33]. 
Table 1. Numerical data for different pressures $\beta P / \kappa^{3}$ : the equilibrium barrier height $\Delta G^{(\mathrm{eq})}$, critical nucleus size $N^{(\mathrm{eq})}$ and second-order corrections to the free energy difference and interfacial free energy as obtained from the fitted simulation data.

\begin{tabular}{llrcc}
\hline$\beta P / \kappa^{3}$ & $\beta \Delta G^{(\mathrm{eq})}$ & $N^{(\mathrm{eq})}$ & $c_{0} D^{2} \kappa^{4}$ & $\kappa_{0} D^{2} \kappa^{4}$ \\
\hline 0.200 & 34 & 209 & $-4.8 \times 10^{4}$ & $6 \times 10^{3}$ \\
0.224 & 21 & 133 & $-4.1 \times 10^{4}$ & $5 \times 10^{3}$ \\
0.240 & 17 & 97 & $-3.4 \times 10^{4}$ & $4 \times 10^{3}$ \\
\hline
\end{tabular}

It is important to realize that the shear rate should not be considered as a thermodynamic variable [32]. In fact, in a recent study of the effect of shear on the location of the solidliquid coexistence in a Lennard-Jones system, Butler and Harrowell [34] found that no purely thermodynamic description of the effect of shear was possible. Shear directly affects the transport of particles from the solid to the liquid phase, and this effect is not thermodynamic. The expansion in equation (7) is simply a way to represent the effect of shear as if it were purely thermodynamic. A priori there is no reason to expect the validity of these assumptions and it is therefore very surprising that this simple extension of classical nucleation theory is able to describe our observations.

\section{The structure and relative orientation of the nucleus}

From an analysis of the bond orientational order parameters it follows that the structure of the nucleus is predominantly body-centred cubic, even though the stable equilibrium phase is the face-centred-cubic crystal. An intriguing question is whether the shape and/or orientation of the solid nuclei that are formed in a supercooled liquid are influenced by the presence of moderate shear rates. Also, without shear, a nucleus is in general neither spherical nor convex. To this end we calculate for each cluster the moment of inertia tensor. After diagonalization, the principal moments of inertia are obtained. For a truly spherical object these should be identical, but due to natural fluctuations this is usually not the case.

In figure 6 we show the average ratios $\lambda_{+} / \lambda_{0}$ and $\lambda_{-} / \lambda_{0}$ of the largest $\left(\lambda_{+}\right)$and smallest $\left(\lambda_{-}\right)$moments of inertia to the intermediate $\left(\lambda_{0}\right)$ one as function of the cluster size $n$. The dependence on the cluster size indicates that larger clusters are slightly more spherical. It is interesting to observe that the shear rates have no visible influence on these ratios, which suggests that the shape does not depend strongly on the applied shear. This is different from the case for the radial distribution functions that we measured in the liquid under shear, as they become increasingly asymmetric for higher shear rates.

The procedure of diagonalization of the moment of inertia tensor also enables us to characterize the orientation of the main axes of the nucleus. Whereas the shape is hardly influenced by the shear, there is a coupling between the orientation of the nucleus and the direction of the shear. To quantify this, we calculate the direction of the largest principal moment (smallest dimension of the nucleus). The $z$-component of the corresponding unit vector gives the cosine of the angle with the vorticity direction. This is weighted with the second Legendre polynomial $P_{2}$ in order to take the symmetries into account. The result is shown in figure 7, where $\left\langle P_{2}(z)\right\rangle$ is plotted versus the cluster size. For an isotropic cluster shape distribution, this correlation function should be zero as is found for the zero-shear case. In the presence of shear, however, a negative correlation is found that grows for larger nuclei. This suggests that the smallest dimension of a nucleus has a preference for being oriented perpendicular to the vorticity direction. 

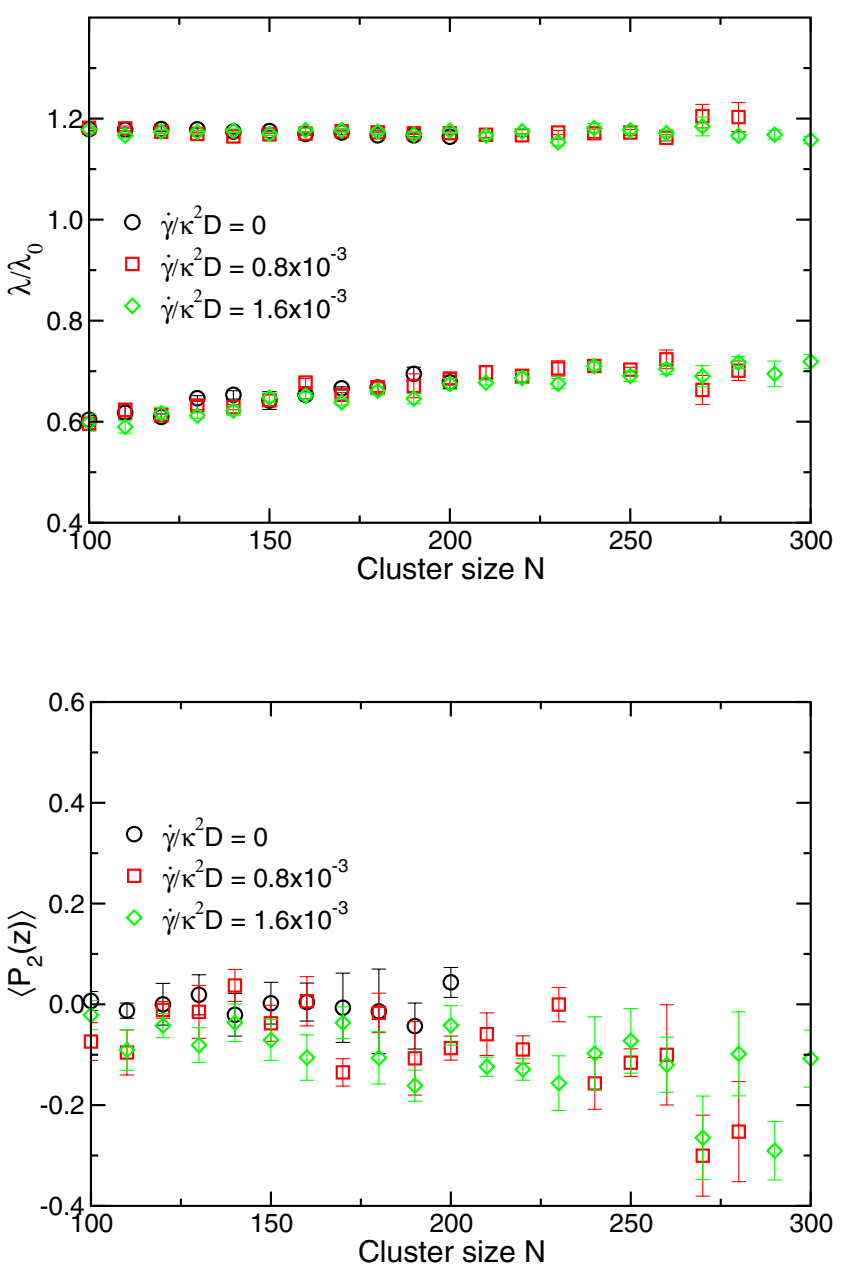

Figure 6. The ratios of the maximum and minimum moments of inertia to the intermediate one as a function of the number of particles in the nucleus and for different shear rates and constant pressure $\beta P / \kappa^{3}=0.2$.

Figure 7. The correlation $\left\langle P_{2}(z)\right\rangle$ of the coupling between the direction of the major moment of inertia and the vorticity direction $\hat{z}$ as a function of the size of the nucleus and for constant pressure $\beta P / \kappa^{3}=0.2$.

In the same manner we can quantify the correlation between the other directions of the principal moments with axes of the reference frame. From this analysis it follows that the largest dimension of the nucleus (smallest moment of inertia) is preferentially along the vorticity direction, while the smallest dimension prefers to be in the plane of the gradient and shear direction. Even within this plane, the distribution of orientations is not isotropic. The measured correlations enable us to calculate the average angle made between the smallest dimension and the gradient direction; the results are shown in figure 8. In order to improve the statistics we have averaged over all clusters containing more than 100 particles up to the size of the critical nucleus $N^{*}$. The inset of figure 8 shows a schematic drawing of the preferred orientation of the nucleus with respect to the reference frame and indicates the tilt angle $\theta$. Note that the largest dimension of the nucleus (smallest principal moment) is preferably along the vorticity direction, i.e. perpendicular to the plane of the drawing.

As can be seen from figure 8, the tilt angle increases linearly with the applied shear rate $\dot{\gamma}$ and only depends weakly on the osmotic pressure. Interestingly, a similar tilt occurs when vesicles with a flexible shape are exposed to a linear shear flow [35]. It is, however, unclear whether this is coincidental or not. 


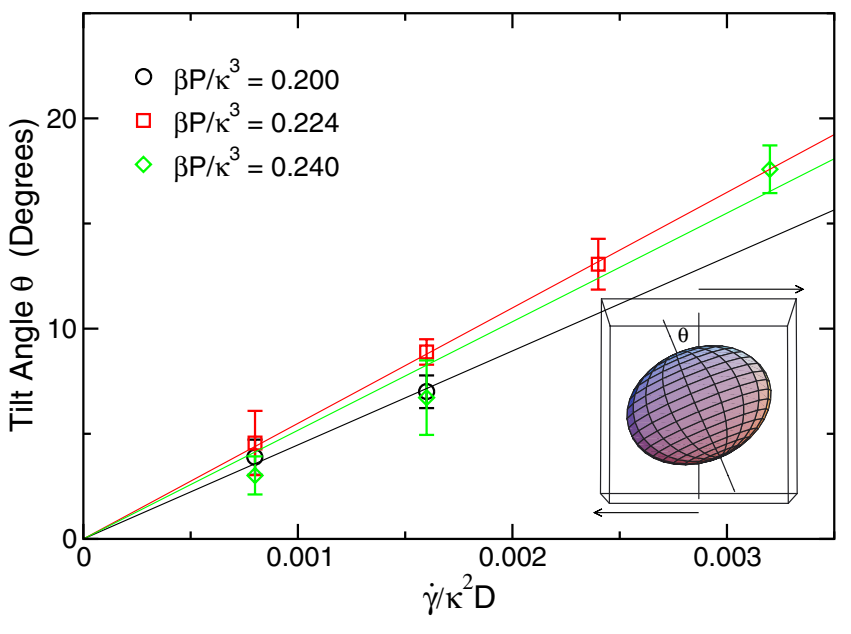

Figure 8. The tilt angle $\theta$ of the principal moment of inertia with respect to the $y$-axis. The inset shows a schematic representation of the preferred orientation of the nucleus with respect to the shear direction indicated by the arrows.

\section{Conclusions}

In summary we have studied the influence of shear on the nucleation of particles with Yukawa interaction. To this end we used a combination of Brownian dynamics simulations with a sort of umbrella sampling technique. The main result is that the probability of crystal nucleation decreases with shear rate, while the critical nucleus size increases. The shape of nuclei is hardly influenced by the presence of moderate shear rates, but it will affect their orientation.

In order to understand the qualitative behaviour of the observations, we used an empirical extension of classical nucleation theory in which we carry over simple equilibrium concepts to a situation of non-equilibrium. A priori there is no reason to expect such a procedure to be valid, but surprisingly it is capable of describing our observations and we hope this will stimulate fundamental research into non-equilibrium physics.

The observations made here could in principle be tested experimentally, by studying homogeneous crystal nucleation in sheared colloidal suspensions. If a Poiseuille flow as realized in a capillary viscometer [36] were to be used, rather than homogeneous Couette flow, we should expect crystal nuclei to appear preferentially in the middle of the flow channel.

For practical reasons we only considered fluids that are weakly sheared, for which one might expect shear-induced layering to be unimportant. One can envisage that for larger shear such pre-ordering phenomena could become relevant, and eventually lead to a situation for which crystal nucleation is enhanced [33]. Another important assumption has been that we ignored hydrodynamic interactions for computational reasons. Although this is allowed for a dilute system, it is in general not correct. Closely related to this is that the implicit solvent is assumed to have a homogeneous linear velocity profile and be unaffected by the presence of the denser crystalline nucleus. For lower pressures and higher shear rates this is not correct [37], as the local transport properties of the liquid can be influenced by the nucleus and consequently affect the nucleation process.

The method can easily be extended to include oscillatory shear [38] or for considering heterogeneous nucleation [39] near a system wall in a sheared suspension. The combination of umbrella sampling with a dynamical simulation method in order to obtain information on rare events is generally applicable in equilibrium and non-equilibrium situations and is not restricted to Brownian dynamics.

Heterogeneous nucleation in a sheared system confined between two parallel plates was studied experimentally by Palberg and co-workers [19] in another paper of this Special Issue. 
A detailed comparison between experiment and theory is in principle possible but is left for future work.

\section{Acknowledgments}

We like to thank T Palberg, A Van Blaaderen, G Szamel and S Egelhaaf for helpful discussions. This work was supported by the Deutsche Forschungsgemeinschaft (DFG), within subproject D1 of the SFB-TR6 programme. The work of the FOM Institute is part of the research programme of the 'Stichting voor Fundamenteel Onderzoek der Materie' (FOM), which is financially supported by the 'Nederlandse organisatie voor Wetenschappelijk Onderzoek' (NWO).

\section{References}

[1] Volmer M and Weber A 1926 Z. Phys. Chem. 119277

[2] Becker R and Döring W 1935 Ann. Phys. 24719

[3] Kelton K F 1991 Solid State Physics vol 45, ed H Ehrenreich and D Turnbull (New York: Academic) pp 75-178

[4] Galkin O and Vekilov P G 2000 Proc. Natl Acad. Sci. USA 976277

[5] Shi F G, Tong H Y and Ayers J D 1995 Appl. Phys. Lett. 67350

[6] Schätzel K and Ackerson B J 1993 Phys. Rev. E 483766

[7] Harland J L and van Megen W 1997 Phys. Rev. E 553054

[8] Sinn C, Heymann A, Stipp A and Palberg T 2001 Prog. Colloid Polym. Sci. 118266

[9] Pusey P 1991 Liquids, Freezing and the Glass Transition ed J P Hansen, D Levesque and J ZinnJustin (Amsterdam: North-Holland) pp 763-942

[10] Gasser U, Weeks E R, Schofield A, Pusey P N and Weitz D A 2001 Science 292258

[11] Auer S and Frenkel D 2001 Nature 4091020

[12] Auer S and Frenkel D 2002 J. Phys.: Condens. Matter 147667

[13] Ackerson B J and Pusey P N 1988 Phys. Rev. Lett. 611033

[14] Yan Y D, Dhont J K G, Smits C and Lekkerkerker H N W 1994 Physica A 20268

[15] Haw M D, Poon W C K and Pusey P N 1998 Phys. Rev. E 576859

[16] Amos R M, Rarity J G, Tapster P R, Shepherd T J and Kitson S C 2000 Phys. Rev. E 612929

[17] Palberg T, Mönch W, Schwarz J and Leiderer P 1995 J. Chem. Phys. 1025082

[18] Okubo T and Ishiki H 1999 J. Colloid Interface Sci. 211151

[19] Stipp A, Biehl R, Preis T, Liu J, Barreira Fontecha A, Schöpe H J and Palberg T 2004 J. Phys.: Condens. Matter $16 \mathrm{~S} 3885$

[20] Butler S and Harrowell P 1995 Phys. Rev. E 526424

[21] Blaak R, Auer S, Frenkel D and Löwen H 2004 Phys. Rev. Lett. 93068303

[22] Allen M P and Tildesley D J 1987 Computer Simulations of Liquids (Oxford: Oxford University Press)

[23] Chakrabarti J, Sood A K and Krishnamurthy H R 1994 Phys. Rev. E 50 R3326

[24] Lees A W and Edwards S F 1972 J. Phys. C: Solid State Phys. 51921

[25] Steinhardt P J, Nelson D R and Ronchetti M 1983 Phys. Rev. B 28784

[26] van Duijneveldt J S and Frenkel D 1992 J. Chem. Phys. 964655

[27] ten Wolde P R, Ruiz-Montero M J and Frenkel D 1995 Phys. Rev. Lett. 752714

[28] Auer S and Frenkel D 2004 J. Chem. Phys. 1203015

[29] O'Malley B and Snook I 2003 Phys. Rev. Lett. 90085702

[30] Torrie G M and Valleau J P 1974 Chem. Phys. Lett. 28578

[31] Hamaguchi S, Farouki R T and Dubin D H E 1997 Phys. Rev. E 564671

[32] Daivis P J and Matin M L 2003 J. Chem. Phys. 11811111

[33] Stevens M J and Robbins M O 1993 Phys. Rev. E 483778

[34] Butler S and Harrowell P 2003 J. Chem. Phys. 1184115

[35] Abkarian M, Lartigue C and Viallat A 2002 Phys. Rev. Lett. 88068103

[36] Palberg T and Würth M 1996 J. Physique 6237

[37] Reguera D and Rubi J M 2003 J. Chem. Phys. 1199888

[38] Xue W and Grest G S 1989 Phys. Rev. A 40 R1709

[39] Auer S and Frenkel D 2003 Phys. Rev. Lett. 91015703 